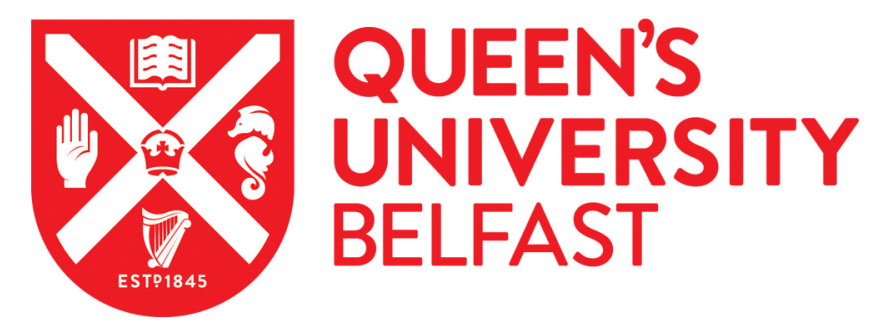

\title{
Revisiting the Schistosoma japonicum life cycle transcriptome for new insights into lung schistosomula development
}

King, M., Carson, J., Stewart, M. T., \& Gobert, G. N. (2021). Revisiting the Schistosoma japonicum life cycle transcriptome for new insights into lung schistosomula development. Experimental Parasitology, 108080. https://doi.org/10.1016/j.exppara.2021.108080

\author{
Published in: \\ Experimental Parasitology
}

Document Version:

Peer reviewed version

Queen's University Belfast - Research Portal:

Link to publication record in Queen's University Belfast Research Portal

\section{Publisher rights}

Copyright 2021 Elsevier.

This manuscript is distributed under a Creative Commons Attribution-NonCommercial-NoDerivs License

(https://creativecommons.org/licenses/by-nc-nd/4.0/), which permits distribution and reproduction for non-commercial purposes, provided the author and source are cited.

\section{General rights}

Copyright for the publications made accessible via the Queen's University Belfast Research Portal is retained by the author(s) and / or other copyright owners and it is a condition of accessing these publications that users recognise and abide by the legal requirements associated with these rights.

Take down policy

The Research Portal is Queen's institutional repository that provides access to Queen's research output. Every effort has been made to ensure that content in the Research Portal does not infringe any person's rights, or applicable UK laws. If you discover content in the Research Portal that you believe breaches copyright or violates any law, please contact openaccess@qub.ac.uk. 
1 Revisiting the Schistosoma japonicum life cycle transcriptome for new

2 insights into lung schistosomula development

3 Meághan King, Jack Carson, Michael T. Stewart and Geoffrey N. Gobert*

4 School of Biological Sciences, Queen's University Belfast, Belfast, BT9 5DL, UK.

$5 \quad *$ Corresponding author, g.gobert@qub.ac.uk

\section{Abstract}

Schistosome parasites are complex trematode blood flukes responsible for the disease schistosomiasis; a global health concern prevalent in many tropical and sub-tropical countries. While established transcriptomic databases are accessed ad hoc to facilitate studies characterising specific genes or gene families, a more comprehensive systematic updating of gene annotation and survey of the literature to aid in annotation and context is rarely addressed. We have reanalysed an online transcriptomic dataset originally published in 2009, where seven life cycle stages of Schistosoma japonicum were examined. Using the online pathway analysis tool Reactome, we have revisited key data from the original study. A key focus of this study was to improve the interpretation of the gene expression profile of the developmental lung-stage schistosomula, since it is one of the principle targets for worm elimination. Highly enriched transcripts, associated with lung schistosomula, were related to a number of important biological pathways including host immune evasion, energy metabolism and parasitic development. Revisiting large transcriptomic databases should be considered in the context of substantial new literature. This approach could aid in the improved understanding of the molecular basis of parasite biology. This may lead to the identification of new targets for diagnosis and therapies for schistosomes, and other helminths.

\section{Keywords}

25 Schistosomiasis; Transcriptomics; Schistosomula; Pathway Analysis; Helminths. 


\section{Introduction}

Schistosomiasis, originally referred to as Bilharzia, is a parasitic disease caused by several species of trematode of the genus Schistosoma. The blood fluke is a zoonotic parasitic helminth, with some species infecting humans and agriculturally important animals (Gordon, et al., 2016). Schistosomiasis affects more than 230 million people worldwide, however there is risk of future infection in an additional 500-779 million individuals (Crompton, 1999, Gryseels, et al., 2006, McManus, et al., 2020). The World Health Organisation (WHO) identifies schistosomiasis as a Neglected Tropical Disease (NTD) which is endemic in 78 tropical and sub-tropical countries (Chitsulo, et al., 2000, Colley and Secor, 2014). Three different strains are predominately responsible for human schistosomiasis; Schistosoma japonicum, S. mansoni, and S. haematobium, however S. japonicum is the main causative agent in Southeast Asia (Gordon, et al., 2015a, Gordon, et al., 2015b).

\section{The Importance of Lung-Schistosomula during the Schistosome Life Cycle}

Parasitic organisms often demonstrate complex life cycles including the requirement of several hosts. Trematodes exploit specific species of freshwater snails, with Oncomelania spp acting as an intermediate host for S. japonicum. Snails release hundreds of free swimming cercariae, which infiltrate the skin of the definitive mammalian host. In the course of host penetration the cercariae activate their development into schistosomula, larvae which will further mature and develop in the host blood stream. The extensive forebody transformation to schistosomula corresponds with intense biochemical and physiological changes to establish their further maturation within the definitive mammalian host (Gobert, et al., 2007, Gobert, et al., 2010). Schistosomula transverse into the blood or lymphatic vessels of the pulmonary circulation, where they will travel to infect the lung. Schistosomula exit the lungs and re-enter the systemic venous system, facilitating migration to the hepatic portal system, were the pre-adult can sexually mature and reside (Gobert, et al., 2007).

The biochemical alterations of the lung schistosomula inherently provide the host immune system with an array of antigens (Wilson, 2009). However, while in the lungs migrating schistosomula are resistant to both adaptive and innate host immune responses (Riengrojpitak, et al., 1998). This successful host-parasite relationship demonstrates the ability of the schistosome to adapt and thrive within the hostile conditions of the host venous system. Published literature highlights possible immune evasion mechanisms (Gobert, et al., 2007, Wilson, 2009), including the rapid 
turnover of the heptalaminate tegument membrane (Gobert, et al., 2003), the secretion of schistosome derived immune modulators (Wilson, 2012), and epitope concealment and antigen masking (Sepulveda, et al., 2010), all of which will contribute to schistosome survival and the down regulation of the host immune response. The lung-stage of the schistosome is the principle phase for potential worm elimination, by host immunological response. Schistosomula could provide insights for possible vaccine targets, due to their endurance regarding the host immune attack (Wilson, 2009).

\section{Previously reported Schistosomula Transcriptomics}

The original study carried out high throughput microarray analysis of the blood fluke S. japonicum, in order to profile genes which were enriched throughout seven differential stages of the schistosome life cycle (Gobert, et al., 2009). At the time, Gene Ontology analysis was carried out enabling the identification of genes involved in developmental changes, and stage specific functions and processes within the parasite; host immune evasion, the acquisition of nutrients, energy production, calcium signalling, sphingolipid metabolism, egg production and tegument function. However, comprehensive pathway analysis was not presented. Due to their biological importance, we now focus on the enriched or upregulated genes in the Day 3 in vivo derived schistosomula as identified in the original study.

Data from the original findings published in 2009 had additional annotation provided and was then mapped to pathways for re-mining, in order to review and update (Gobert, et al., 2009). This included the use of supplementary tables in the publication and GEO Gene Expression Omnibus, http://www.ncbi.nlm.nih.gov/geo/ accession numbers GPL7160, GSE12704. All data was filtered for all of the 19,222 contigs previously present on the schistosome microarray. Signals that were flagged "present" during the feature extraction process were included, as well as p-values (ANOVA with multiple testing correction: Benjamini and Hochberg False Discovery Rate) $\leq 0.05$. Finally an arbitrary $\geq 2$ fold up-regulation relative to the mean fold intensity of combined life cycle stages, was used to identify lung schistosomula enriched genes.

\subsection{Updated annotation and Reactome pathway analysis online database}

All genes were subjected to DIAMOND BLASTX, a heuristic open source algorithm able to translate contig nucleotide sequences into viable and accurate protein description, using a protein database. BLASTX, often referred to as the gold standard tool for offering such high sensitivity, is much too 
slow for application of such a large nucleotide sequence dataset. Appropriate for multiple sequences, long read analysis and capable of translation speeds 20,000 times faster than BLASTX alone, the DIAMOND sequence aligner was selected to provide high sensitivity of large data input within a much shorter time period (Buchfink, et al., 2015). The closest human homologue was identified, with accession numbers used for subsequent Reactome analysis.

Reactome is an online (https://reactome.org/) curated database, representative of human biological pathways and reactions (Fabregat, et al., 2018). It provides an in-built bioinformatics tool for the interpretation of pathway analysis through visual representation. Along with protein description and gene ontology (GO), BLASTX analysis also provided accession numbers, required by Reactome. It is not a specialised bioinformatics tool for parasites. As a result, contig nucleotide sequences must be converted to the closest human homologue. Analysis was performed against Reactome version 68. Human homologue accession numbers were then compared to genes upregulated within human pathways to identify relevant biological pathways. Pathways were organised in order of significance as determined by p-value (Fabregat, et al., 2017) and the pathway hierarchy was visualised through Reactome, which allowed for the final identification of genes expressed in relation to key aspects of lung-stage schistosomula biology.

The Reactome database incorporates a species comparison tool, enabling comparative analysis between human pathways and those predicted in the model organism Caenorhabditis elegans (Haw and Stein, 2012, Fabregat, et al., 2018). Widely used for research in developmental biology, there is extensive knowledge of its biology and genome, C. elegans is very well characterised (Duguet, et al., 2020). Therefore, a comparison between the free-living nematode; C. elegans, and the parasitic platyhelminth S. japonicum can be used to reveal the molecular basis of parasitism. C. elegans and S. japonicum through releasing extracellular vehicles (EVs) may play a vital role in both organisms and aspects of their behaviour (de la Torre-Escudero, et al., 2019, Duguet, et al., 2020). In this context, using the model organism as a comparator was beneficial in terms of developmental biology and host-parasite interactions. Comparison between $C$. elegans and S. japonicum to reveal the basis of parasitism may be problematic. Any significant transcriptional differences between the two organism may not be related to parasitism. Since one is a nematode and the other a platyhelminth, these represent species relatively diverged in evolution. Therefore, differences may be associated with physiological characteristics instead of parasitism. Comparison with a free-living platyhelminth (Martin-Duran and Egger, 2012) may provide stronger indicators of parasitism. 
Re-annotation and re-analysis of the gene expression data was extremely advantageous, in the context of the substantial new literature published following 2009 (Gobert, et al., 2009). When compared to GO and KEGG pathway analysis, Reactome can provide a better way to interpret and visualise data. Reactome is however not a specialised bioinformatics tool for helminths. Nucleotide sequences must be matched to human homologues, as such analysis may be influenced by this reliance on human databases. For example topics such as the immune system, may be over represented.

\subsection{Data subset for reanalysis and reinterpretation}

125 A gene list containing 1,311 contigs upregulated in the lung schistosomula was generated, including 126 the removal of any technical duplicates (see Supplementary Table 1). The 10 most up-regulated transcripts by relative fold change, are presented in Table 1. Examples of enriched lung schistosomula genes included cytochrome c oxidase subunit iii (Contig4489 2,805.95 fold) and eukaryotic translation elongation factor 1 alpha (Contig560 533.95 fold).

\section{Pathway Analysis}

Reactome analysis of enriched genes present in the lung schistosomula, mapped pathways for 728 of the 1,311 identifiers (genes) in the data. The transcriptome wide pathway overview (Fig. 1) enabled the hierarchical visualisation of generated pathways, biologically important to the lungstage schistosomula (Fabregat, et al., 2017). Reactome analysis was able to depict the ten most significant pathways (Table 2), determined by $p$-value. The most significant pathway identified was the Endosomal/Vacuolar pathway, with a $p$-value $=1.11 \mathrm{E}-16$. The 5 most significant pathways are all related to functions within the immune system. 

et al., 2009.

\begin{tabular}{llcc}
\hline Contig/Probe & Nucleotide Description/Protein Homology & $\boldsymbol{p}_{\text {-value }} \mathbf{1}^{\mathbf{1}}$ & Fold Change $^{\mathbf{2}}$ \\
\hline TC17941 & ferritin heavy chain & $1.10 \mathrm{E}-04$ & 12231.52 \\
TC14376 & Zinc phosphodiesterase ELAC protein 2 & $5.70 \mathrm{E}-04$ & 5701.36 \\
Contig4489 & cytochrome c oxidase subunit iii & $1.42 \mathrm{E}-04$ & 2805.95 \\
TC19335 & Tetratricopeptide repeat protein 25 & $1.44 \mathrm{E}-03$ & 1273.01 \\
TC11085 & T cell receptor beta variable 5-8 & $5.92 \mathrm{E}-04$ & 1240.04 \\
Contig6759 & Immunoglobulin superfamily member 10 (IgSF10) CMF608 & $1.07 \mathrm{E}-03$ & 1206.84 \\
TC12767 & N-acetylglucosamine-1-phosphotransferase subunits GNPTAB & $1.87 \mathrm{E}-03$ & 982.79 \\
TC15743 & Alpha-2,8-sialyltransferase 8B & $1.91 \mathrm{E}-03$ & 971.16 \\
Contig560 & eukaryotic elongation factor 1 alpha & $9.83 \mathrm{E}-04$ & 533.95 \\
TC7263 & Cystic fibrosis transmembrane conductance regulator (CFTR) & $1.35 \mathrm{E}-03$ & 512.88 \\
\hline
\end{tabular}

${ }^{2}$ Fold Change is relative to the average expression of all seven S. japonicum life cycles stages examined.

Table 2. The ten most significant Reactome pathways enriched in lung schistosomula determined by $p$ value.

\begin{tabular}{lcccc}
\hline Pathway Name & Entities found & Ratio & P-value & FDR* \\
\hline Endosomal/Vacuolar pathway & $36 / 82$ & 0.004 & $1.11 \mathrm{E}-16$ & $2.05 \mathrm{E}-13$ \\
Antigen Presentation Folding, assembly and peptide loading & $44 / 217$ & 0.011 & $9.20 \mathrm{E}-07$ & $7.00 \mathrm{E}-04$ \\
of class I MHC & $49 / 298$ & 0.015 & $1.30 \mathrm{E}-06$ & $7.00 \mathrm{E}-04$ \\
ER-Phagosome pathway & $51 / 320$ & 0.016 & $1.52 \mathrm{E}-06$ & $7.00 \mathrm{E}-04$ \\
Antigen processing-Cross presentation & $56 / 480$ & 0.024 & $4.82 \mathrm{E}-05$ & 0.018 \\
Neutrophil degranulation & $20 / 119$ & 0.006 & $1.92 \mathrm{E}-04$ & 0.059 \\
SRP-dependent cotranslational protein targeting to & $18 / 110$ & 0.005 & $5.30 \mathrm{E}-04$ & 0.139 \\
membrane & $76 / 670$ & 0.033 & 0.003 & 0.606 \\
Formation of a pool of free 40S subunits & $9 / 45$ & 0.002 & 0.004 & 0.646 \\
Class I MHC mediated antigen processing \& presentation & $16 / 111$ & 0.006 & 0.004 & 0.646 \\
RHO GTPases activate IQGAPs & & & & \\
Eukaryotic Translation Termination & &
\end{tabular}

* False Discovery Rate.

148 Due to the large quantity of data demonstrating function within the immune system, pathways were 149 further analysed using a hierarchy overview. Results reveal a pathway hierarchy, each step away 150 from the centre will represent a level lower in the hierarchy. The highest level pathway is Class I 151 MHC mediated antigen processing \& presentation, projected outwards from the central node of the 152 immune system (Fig. 2A). Lower level pathways were also identified as shown in Fig. 2b. Reactome 

analysis reports identified individual accession numbers responsible for pathway generation. The data reveals numerous genes which contribute to several different pathways related to immune function. For example, the 26S proteasome non-ATPase subunit RPN5 (Contig7591) was detected in four different pathways; ER-Phagosome pathway, Antigen processing-Cross presentation, Antigen processing: ubiquitination \& proteasome degradation and Cross presentation of soluble exogenous antigens (Endosomes) (Supplementary Table 2).

Supplementary Table 2. Examples of genes contributing to immune pathways.

\begin{tabular}{|c|c|c|c|c|}
\hline Pathway & Genes & Contig & $p$ value ${ }^{1}$ & $\begin{array}{c}\text { Fold } \\
\text { Change }^{2}\end{array}$ \\
\hline \multirow[t]{3}{*}{ Endosomal/Vacuolar pathway } & HLA class I antigen $\mathrm{Cw}-7 \alpha$-chain & TC14680 & 1.10E-02 & 15.87 \\
\hline & HLA class I antigen A- $3 \alpha$-chain & Contig8280 & 2.77E-02 & 5.09 \\
\hline & Cathepsin L & Contig1966 & $3.83 \mathrm{E}-02$ & 2.78 \\
\hline \multirow{2}{*}{$\begin{array}{l}\text { Antigen Presentation: Folding, } \\
\text { assemble and peptide loading } \\
\text { of class I MHC }\end{array}$} & HLA class I antigen, $\mathrm{C} w-7 \alpha$-chain & TC14680 & $1.10 \mathrm{E}-02$ & 15.87 \\
\hline & HLA class I antigen, A- $3 \alpha$-chain & Contig8280 & 2.77E-02 & 5.09 \\
\hline \multirow[t]{6}{*}{ ER-Phagosome pathway } & Proteasome subunit $\alpha$ - 8 & TC13609 & 4.01E-03 & 16.97 \\
\hline & $26 \mathrm{~S}$ proteasome non-ATPase subunit RPN5 & Contig7591 & $1.04 \mathrm{E}-02$ & 11.26 \\
\hline & $26 \mathrm{~S}$ proteasome non-ATPase subunit RPN8 & TC10760 & $2.90 \mathrm{E}-03$ & 10.59 \\
\hline & $26 \mathrm{~S}$ proteasome non-ATPase subunit RPN5 & Contig7514 & $2.63 \mathrm{E}-02$ & 7.58 \\
\hline & $26 \mathrm{~S}$ proteasome non-ATPase subunit RPN1 & Contig780 & $1.03 \mathrm{E}-02$ & 5.31 \\
\hline & Proteasome subunit $\beta-5$ & TC10632 & 1.67E-02 & 5.16 \\
\hline \multirow{4}{*}{$\begin{array}{l}\text { Antigen processing-Cross } \\
\text { presentation }\end{array}$} & Proteasome subunit $\alpha-8$ & TC13609 & 4.01E-03 & 16.97 \\
\hline & $\begin{array}{l}26 \mathrm{~S} \text { proteasome non-ATPase subunits } \\
\text { RPN5,8,1 }\end{array}$ & & $\begin{array}{l}\text { See } \\
\text { Above }\end{array}$ & \\
\hline & Proteasome subunit $\beta-5$ & TC10632 & 1.67E-02 & 5.16 \\
\hline & Cathepsin L & Contig1966 & $3.83 \mathrm{E}-02$ & 2.78 \\
\hline \multirow{3}{*}{$\begin{array}{l}\text { Antigen processing: } \\
\text { ubiquitination \& proteasome } \\
\text { degradation AND } \\
\text { Cross presentation of soluble } \\
\text { exogenous antigens } \\
\text { (Endosomes) }\end{array}$} & Proteasome subunit $\alpha-8$ & TC13609 & $4.01 E-03$ & 16.97 \\
\hline & $\begin{array}{l}26 \mathrm{~S} \text { proteasome non-ATPase subunits } \\
\text { RPN5,8,1 }\end{array}$ & & $\begin{array}{l}\text { See } \\
\text { Above }\end{array}$ & \\
\hline & Proteasome subunit $\beta-5$ & TC10632 & 1.67E-02 & 5.16 \\
\hline $\begin{array}{l}\text { Cross presentation of } \\
\text { particulate exogenous } \\
\text { antigens (Phagosomes) }\end{array}$ & Integrin $\beta-5$ & Contig2213 & $1.44 \mathrm{E}-02$ & 25.13 \\
\hline
\end{tabular}

$163{ }^{2}$ Fold Change is relative to the average expression of all seven S. japonicum life cycles stages examined. 


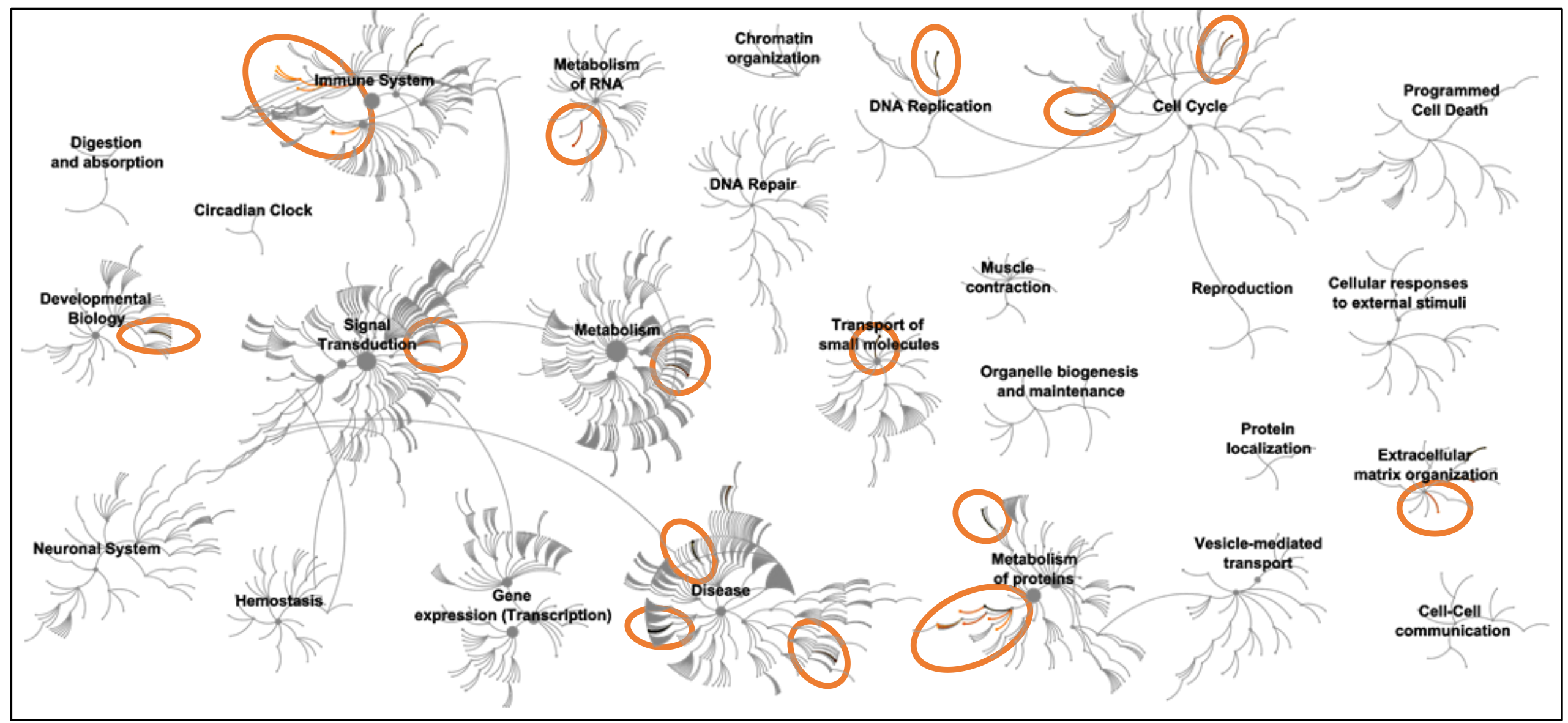

Fig. 1. Overview of Reactome pathway analysis of lung schistosomula enriched genes. The pathways are arranged hierarchically, with the centre of each circular "burst" representing the root of a top level pathway, such as "Immune System". Each stride away from the centre signifies a lower level in the hierarchy of pathways. The circled areas indicates a pathway(s) that is over-represented in the dataset. Grey denotes pathways which are not significantly over-represented during pathway analysis. 


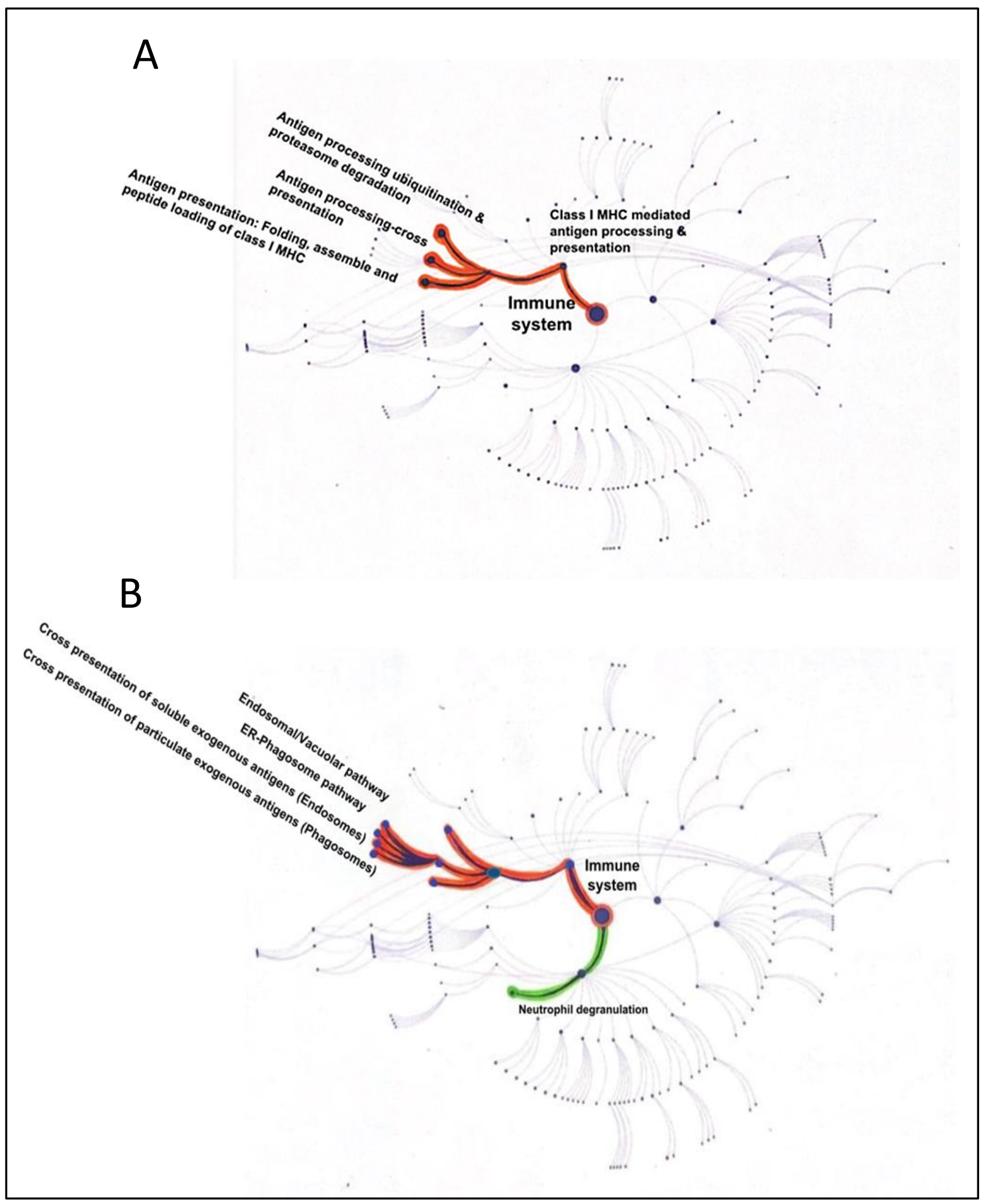

Fig. 2. Hierarchy overview of high level pathways observed in the immune system. A. Class $1 \mathrm{MHC}$ mediated antigen processing \& presentation was identified as the highest level pathway, followed by Antigen processing ubiquitination \& proteasome degradation, Antigen processing-cross presentation and Antigen presentation: Folding, assemble and peptide loading of class I MHC. B. The endosomal/vascular pathway, ER-Phagosome pathway, Cross presentation of soluble exogenous antigens (Endosomes) and Cross presentation of particulate exogenous antigens (Phagosomes), represent the lowest level pathways within the immune system. The highlighted regions are representative of innate and adaptive immunity, green (highlighted central to down in B.) signifies 
a unit of the innate immune pathway including neutrophil degranulation. Orange (all highlights in A. and central to left highlights in B) is representative of adaptive immune pathways, involving antigen processing and presentation.

Neutrophil degranulation (Fig. 2B) was found to be a significant pathway within the lung-stage schistosomula (Table 2), with 64 genes contributing to the pathway. Novel genes of interest were identified (Supplementary Table 3), including Elongation factor 1-alpha (Contig560) with a large fold change of 533.95. Elongation factor 1-alpha is also one of the top 10 most upregulated transcripts in the data set, shown above in Table 1. An overview of the immune system demonstrates different mechanisms of relevance, such as those involved in Innate and Adaptive Immunity (Fig. 2B).

Supplementary Table 3. Selected lung schistosomula enriched genes associated within the neutrophil degranulation pathway.

\begin{tabular}{lccc}
\hline Gene & Contig & $\boldsymbol{p}$ value $^{\mathbf{1}}$ & Fold Change $^{2}$ \\
\hline Eukaryotic elongation factor 1 alpha & Contig560 & $9.83 \mathrm{E}-04$ & 533.95 \\
Secretory carrier-associated membrane protein & TC8138 & $1.19 \mathrm{E}-02$ & 85.29 \\
Tetraspanin & TC18168 & $1.39 \mathrm{E}-02$ & 72.23 \\
Endoplasmic reticulum resident protein & Contig3800 & $2.72 \mathrm{E}-02$ & 38.07 \\
Vesicle-associated membrane protein & TC13962 & $5.03 \mathrm{E}-03$ & 18.79 \\
Cathepsin D & Contig6337 & $5.86 \mathrm{E}-03$ & 14.99 \\
26S proteasome non-ATPase subunit RPN5 & Contig7591 & $1.04 \mathrm{E}-02$ & 11.26 \\
26S proteasome non-ATPase subunit RPN8 & TC10760 & $2.90 \mathrm{E}-03$ & 10.59 \\
Tetraspanin cd53 antigen & Contig5336 & $5.54 \mathrm{E}-03$ & 9.09 \\
26S proteasome non-ATPase subunit RPN5 & Contig7514 & $2.63 \mathrm{E}-02$ & 7.58 \\
26S proteasome non-ATPase subunit RPN1 & Contig780 & $1.03 \mathrm{E}-02$ & 5.31 \\
annexin a2a & Contig7025 & $9.94 \mathrm{E}-03$ & 4.57 \\
Cathepsin A & Contig7606 & $5.18 \mathrm{E}-03$ & 4.57 \\
Heat shock cognate 71 protein (cDNA FL77848) & Contig7768 & $3.02 \mathrm{E}-02$ & 3.81 \\
TGF beta receptor associated protein -1 & TC19148 & $4.67 \mathrm{E}-02$ & 3.64 \\
Cathepsin B & Contig8688 & $7.76 \mathrm{E}-03$ & 3.44 \\
Cathepsin L & Contig1966 & $3.83 \mathrm{E}-02$ & 2.78 \\
\hline
\end{tabular}

192 193

${ }^{1}$ ANOVA with multiple testing correction.

${ }^{2}$ Fold Change is relative to the average expression of all seven S. japonicum life cycles stages examined.

Lists of highly expressed genes that are involved in Energy metabolism, Immune evasion and Development are presented in Supplementary Table 4. Several genes are shown to contribute to different functions, such as Proteasome subunit alpha-type 8 (TC13609), a subunit of the Ubiquitin 
Proteasome Pathway; with a fold change of 16.97, it has been shown to function in several pathways relating to both immune evasion and development. Nutritional transporters are shown to be highly expressed in the lung-stage schistosomula, such as the ATP-binding cassette (ABC) type transporter AbcG6 (TC12977), upregulated at a fold change of 103.86 .

Supplementary Table 4. Selected novel highly enriched genes, associated with Nutritional metabolism, Proteasome degradation/Immune evasion and Development within the lung-stage schistosomula.

\begin{tabular}{llccc}
\hline Function & Gene & Contig & $\boldsymbol{p}$ value & Fold Change $^{2}$ \\
\hline Energy & ABC transporter AbcG6 & TC12977 & $2.61 \mathrm{E}-02$ & 103.86 \\
& Glucose transporter protein (SGTP4) & Contig4074 & $4.78 \mathrm{E}-02$ & 2.45 \\
& Pepsin A-3 & TC13201 & $1.63 \mathrm{E}-02$ & 25.51 \\
& Cathepsins A, B, D, L & & See Table 4 & \\
\hline Immune & Proteasome subunit $\alpha-8$ & TC13609 & $4.01 \mathrm{E}-03$ & 16.97 \\
evasion & 26S proteasome non-ATPase subunits RPN1,5,8 & & See Table 4 & \\
& Proteasome subunit $\beta$-5 & TC10632 & $1.67 \mathrm{E}-02$ & 5.16 \\
& HLA class I antigen CW-7 $\alpha$-chain & TC14680 & $1.10 \mathrm{E}-02$ & 15.87 \\
& HLA class I antigen A-3 $\alpha$-chain & Contig8280 & $2.77 \mathrm{E}-02$ & 5.09 \\
& Eukaryotic elongation factor 1-alpha & Contig560 & $9.83 \mathrm{E}-04$ & 533.95 \\
& Tetraspanin & TC18168 & $1.39 \mathrm{E}-02$ & 72.23 \\
& Tetraspanin cd53 antigen & Contig5336 & $5.54 \mathrm{E}-03$ & 9.09 \\
& Endoplasmic reticulum resident protein & Contig3800 & $2.72 \mathrm{E}-02$ & 38.07 \\
\hline Development & Annexin & TC12719 & $1.00 \mathrm{E}-02$ & 17.33 \\
& Annexin a2a & Contig7025 & $1.00 \mathrm{E}-02$ & 4.57 \\
& Paramyosin & Contig7928 & $1.61 \mathrm{E}-02$ & 18.60 \\
& Proteasome subunit $\alpha-8$ & TC13609 & $4.01 \mathrm{E}-03$ & 16.97 \\
& 26S proteasome non-ATPase subunits RPN1,5,8 & & See Table 4 & \\
& Proteasome subunit $\beta-5$ & TC10632 & $1.67 \mathrm{E}-02$ & 5.16 \\
\hline
\end{tabular}

${ }^{1}$ ANOVA with multiple testing correction.

${ }^{2}$ Fold Change is relative to the average expression of all seven S. japonicum life cycles stages examined.

Out of the 1,311 enriched contig nucleotide sequences subject to Reactome analysis, only 728 identifiers (genes) were mapped to appropriate pathways. Owing to many regions of the $S$. japonicum genome remaining uncharacterised with unknown functions, a large proportion of the Schistosoma transcriptional picture is incomplete. Consequently, more research in functional genomics, including gene knock-out, would be extremely beneficial to improve this situation.

\section{New interpretations}


The new annotations for genes enriched during the lung schistosomula stage were updated and placed in broad biological relevance, using both pathway analysis and literature primarily published since the original study (Gobert, et al., 2009), when the transcriptomics was originally generated, analysed, interpreted and discussed. In the original paper, 1,782 genes were analysed using the bioinformatics platform Blast2Go, providing functional annotation in gene ontology (GO) and KEGG pathway analysis. During the schistosomula lung-stage, gene function was catalogued into two of three main categories modulated during development; biological process and molecular function. Genes enriched within the GO category of biological processes were found to be heavily involved in metabolism (Gobert, et al., 2009). In the present study, we were able to confirm a correlation between the microarray data and prominent GO; metabolic process. Protein metabolism pathways; SRP-dependent co-translational protein targeting to membrane, formation of a pool of free $40 \mathrm{~S}$ subunits and Eukaryotic Translational Termination, were all significant Reactome pathways enriched within the lung schistosomulum (Table 2).

Reactome analysis has identified new pathways containing multiple genes involved in the immune system. Originally, the GO term immune system was not found to be enriched amongst genes upregulated in the lung-stage, compared to all other life cycle stages. However, results did highlight the GO protein binding within the molecular function category and during the present re-annotation, eukaryotic elongation factor-1-alpha (eEF1 $\alpha$ ) was identified as a highly enriched transcript (Table 1).

\section{1 eEF1 $\alpha$ - a potential regulator for apoptosis and host immunomodulation}

Eukaryotic elongation factor 1-alpha (eEf1 $\alpha$ ) (Contig560) was revealed to be one of the most highly upregulated gene transcripts involved in the developmental lung-stage of the schistosome. Reactome pathway analysis also placed this upregulated gene within the neutrophil degranulation pathway. Conserved in many organisms, eEf1 $\alpha$ plays a central role in eukaryotic protein synthesis (Billaut-Mulot, et al., 1994). Functionally, eEF1 $\alpha$ is an enzyme responsible for the catalysis of the GTP-dependent binding of aminoacylated-tRNAs to the A site of the ribosome (Timm, et al., 2017, Wang, et al., 2017). However, eEf1 $\alpha$ also plays a key role in functions other than translation, including apoptosis (Alves, et al., 2015).

The schistosome is a multicellular parasite known to induce apoptosis in host cells (DosReis and Barcinski, 2001, Chen, et al., 2002). Genomic studies have revealed that there are similarities in the 
apoptotic processes present between schistosomes and higher organisms (Schistosoma japonicum Genome Sequencing Functional Analysis Consortium, 2009, Peng, et al., 2010, Peng, et al., 2011, Han, et al., 2013). Features of apoptosis, such as morphological change and condensed chromatin, were characteristic of schistosomula extracted from the resistant mammalian host Microtus fortis (Peng, et al., 2011). The expansion of research concerning the schistosome genome has recognised apoptosis as a vital process, for their growth and host-parasite interactions in each developmental stage of the life cycle (Chen, et al., 2002, Schistosoma japonicum Genome Sequencing Functional Analysis Consortium, 2009, Peng, et al., 2011).

Our reanalysis reveals that eEF1 $\alpha$ was highly expressed in lung schistosomula. The function of eEF1 $\alpha$ as a selective apoptotic regulator has been noted in the unicellular parasitic organism Trypanosoma cruzi, however, there is limited information concerning the contribution of schistosome eEF1 $\alpha$ with regards to apoptotic mechanisms (Billaut-Mulot, et al., 1994, Alves, et al., 2015). eEF1 $\alpha$ proteins can be found in secreted exosome vesicles of S. japonicum (Zhu, et al., 2016) and T. cruzi (Bayer-Santos, et al., 2013). Extracellular vesicles (EV) are known to play an important role in parasite-host interactions (de la Torre-Escudero, et al., 2019). eEF1 $\alpha$ is a pleiotropic protein also involved in noncanonical function, such as apoptotic modulation (both pro- and anti-) through the presence of eEF1 1 and eEF1 $\alpha 2$ isoforms (Abbas, et al., 2015). Parasite survival through the release of an antiapoptotic signal, which blocks apoptosis induced by host cells would be advantageous, in this case to the lung-stage schistosomula. This may promote immune evasion by the schistosomula and immunomodulation of the host response, during a time of increased schistosome development.

Parasitic pathogens have developed strategies to regulate the host immune response to enable immune evasion (Maizels, et al., 2018). The membranes of the schistosome tegument are enriched in abundant phospholipid classes such as phosphatidylcholine (PC) (Retra, et al., 2015), which has also been identified in the S. mansoni lipidome (Giera, et al., 2018). Leishmania infantum are able to form PC-epitopes through the active modification of the eEF1 $\alpha$ protein (Timm, et al., 2017). $L$. infantum eEF1 $\alpha$ was further confirmed to be significant with regards to immunosuppression and priming of the host cells in order to facilitate Leishmania attack (Babu and Nutman, 2019). Our reanalysis and the supported literature has identified eEF1 $\alpha$ protein as a potential virulence factor within the lung-schistosomula. It is also possible that exosome vesicles present on the developing tegument of $S$. japonicum contain eEF1 $\alpha$ modified with PC. PC modified antigen molecules may have an important role in the degradation of host protective cells, through the introduction of eEF1 $\alpha$ and 
its ability to regulate apoptosis; this would disrupt attempts by the host to exterminate the worm or potentially cultivate and acquire a protective response (Jenkins, et al., 2005).

The modification of biomolecules, such as eEF1 $\alpha$, may contribute to the immunomodulatory capacity of the schistosomula. PC-bearing antigens have been found to intervene with host immune cells, potentially interfering with crucial signalling pathways of both B and T cells, dendritic cell maturation and mast cell degranulation. This would enable the schistosome to accomplish immunological resistance to the host and ensure schistosomula survival during the lung-stage of their life cycle. Our hypothesis supports initial claims that lung schistosomula protect themselves through inducing changes in their membrane surface together with the confinement of antigen molecules in lipid-rich sites (El Ridi, et al., 2003, Fonseca, et al., 2012). The presence of eEF1 $\alpha$ as an apoptotic regulator would provide an immunological barrier in addition to a physical obstruction provided by membrane alterations, such as PC modification. Through the use of Reactome, we could better appreciate and visualise eEF1 $\alpha$ as a complex and pleiotropic moonlighting protein subunit (Jakobsson, et al., 2018) implicated in actin-binding (Demma, et al., 1990, Yang, et al., 1990), apoptotic regulation (Abbas, et al., 2015), host immunomodulation and host immune evasion, in addition to its role in protein synthesis.

\subsection{Nutrient Transporters-Schistosome Glucose Transporter Proteins}

Schistosomes within the blood vessels of the mammalian host can transport glucose and other nutrients across their outer tegument membrane (Skelly, et al., 1998, Gobert, et al., 2003). This involves the presence of schistosome glucose transport proteins (SGTP) which are host-exposed and distinctively localised to the tegument of the adult schistosome (Krautz-Peterson, et al., 2010). Our reanalysis revealed the upregulated expression of several genes involved in energy metabolism. The upregulated expression of the gene for SGTP4 was expected due to the presence of the schistosomula heptalaminate tegument, commonly associated with SGTP4. SGTP1 is also expressed in schistosomula, however the gene was not present within our data set as it was $<2$ fold enriched.

RNAi studies by Krautz-Peterson et al. (2010) proposed that glucose demands are higher for in vivo parasites, since SGTP-knockdown parasites displayed less viability in vivo following the infection of mice. It was argued that in order to migrate through host tissues, and in occupying the vasculature and fighting the host immune response, it is vital for the parasite to generate more energy (KrautzPeterson, et al., 2010). Our reanalysis supports this theory, as does the upregulated gene expression of SGTP4 in in vivo lung schistosomula (Gobert, et al., 2009). 


\subsection{Nutrient Transporters-Efflux Transporters}

306 The ATP-binding cassette (ABC) type transporter AbcG6 (TC12977), an efflux transporter that 307 translocates compounds across the tegumental membrane (Greenberg, 2014), was shown in our 308 reanalysis to be highly enriched within lung schistosomula. The presence of transporter molecules is essential for parasitic survival (Fonseca, et al., 2012), and lung schistosomula may potentially have a greater requirement for membrane transporters compared to mature adults (Gobert, et al., 2007). The distinction in tegument thickness, an apparent lack of gut function and the absence of a large absorptive surface may suggest that the schistosomula are not fully functional when equated to the adult parasite regarding nutrition. There are opposing views concerning the lung-stage schistosomula and their ability to ingest host blood; some studies claim that a functional gut is absent within the lung schistosomula, however other research suggests that it is present and could be partially functional (Bennett and Caulfield, 1991, El Ridi, et al., 2003). The upregulated expression of transporter genes could enable schistosomula to thrive without a functional gut, through the direct importation of sugar and fatty acids across the tegument (Krautz-Peterson, et al., 2007, Krautz-Peterson, et al., 2010, Da'dara, et al., 2012).

\subsection{Proteases and schistosomula nutrition}

Proteases, including cysteine and aspartic proteases, are a group of enzymes capable of the catalytic hydrolysis and digestion of peptide bonds. Multiple functions within the schistosome have been proposed including haemoglobin digestion and host invasion (Skelly, et al., 2014, Tan-ariya, et al., 2014). Our reanalysis reveals the upregulated expression of several classes of proteolytic endopeptidase enzymes; cathepsins D (Contig6337), B (Contig8688) and L (Contig1966) in lungstage schistosomula.

Schistosome cathepsin B, cathepsin L and papain-superfamily lysosomal cysteine endopeptidases were upregulated within lung-stage schistosomula, potentially supporting parasite nutrition by the digestion of host haemoglobin released from ingested host erythrocytes (El Ridi, et al., 2014). Cysteine proteases are regulated proteolytic enzymes, essential for schistosome survival and growth within its developmental intra-mammalian stages (Zerda, et al., 1988), due to their association with the invasion of host tissues. The current reanalysis correlates with further transcriptomic studies by Gobert et al. (2010). In the presence of red blood cells, in vitro schistosomula genes encoding cathepsin B and cathepsin L were upregulated greatly in S. mansoni (Gobert, et al., 2010). Gene 
silencing studies have exhibited the inability of schistosomes to feed on host haemoglobin in vitro and failure to survive in vivo without the presence of the proteolytic enzymes cathepsin $B$ and cathepsin L (Correnti, et al., 2005).

Cathepsin D (Contig6337) is an aspartic proteinase also shown to be upregulated within the reanalysed data. The proteolytic enzyme is expressed in the gut of the adult parasite similar to cysteine proteinases and has been shown to be critical in haemoglobin digestion and therefore the survival of the schistosome (Figueiredo, et al., 2015). S. mansoni schistosomula, when subjected to RNAi against cathepsin D (Morales, et al., 2008), exhibited reduced growth in vitro and a retardation of gut function. Furthermore, Cathepsin D knockdown schistosomes were not able to survive following injection into a mouse host (Morales, et al., 2008). In vitro the cathepsin $D^{-}$schistosomula gut appeared red in colour; this is in distinct contrast with the black coloured gut of the controls which contain hematin, the final product of haemoglobin proteolysis (Toh, et al., 2010, Toh, et al., 2015). It was hypothesised that the red colour was due to human haemoglobin, which had not been digested as a result of cathepsin D gene silencing (Morales, et al., 2008).

Our reanalysed data and previous studies both suggest that proteinases are essential for functions in the overall maintenance and survival of schistosomes, particularly schistosomula (Morales, et al., 2008 , Gobert, et al., 2010). The upregulated expression of cathepsins within our reanalysis provides further support for cathepsins important role in nutrition, indicating that lung schistosomula have a functional gut and therefore the ability to feed from the blood stream of the host.

\section{Future prospects}

Traditionally, DNA microarrays have been used to answer a wide range of questions as the standard application for gene expression profiling in Schistosoma genomics and transcriptomics. Microarrays are a reliable and well-established technology that can still provide new insights into biological processes under investigation. Nevertheless, it is an older technology and its limitations still remain omitting RNA-editing events and exon-exon splice junctions for novel expressed regions, and only profiling predefined genes; available from the $S$. japonicum genome and transcriptome, through hybridization (Malone and Oliver, 2011).

The advent of high-throughput sequencing technology in recent years and general advancements in the field of transcriptomics have enabled Wangwiwatsin et al. to investigate, and describe the developmental time-course of $S$. mansoni, with particular emphasis on the lung-stage 
(Wangwiwatsin, et al., 2020). The study includes the first description of RNA-sequencing (RNA-seq) transcriptome analysis on day- 6 in vivo derived lung schistosomula, when 864 genes were found to be upregulated in comparison to day-13 juveniles (Wangwiwatsin, et al., 2020). In contrast, our hybridization-based method was able to generate a gene list of 1,311 identifiers upregulated during the lung-stage. Compared to the original microarray study (Gobert, et al., 2009), this level of analysis is based on a more mature and streamlined genomic structure, enabling a highly sensitive investigation of gene expression. RNA-seq reads can be directly mapped to the S. mansoni genome, which has been collectively re-annotated more than the $S$. japonicum microarray data (Gobert, et al., 2009).

Wangwiwatsin and colleagues also expand on gene ontology enrichment (GO), highlighting the GO term; iron homeostasis, enriched amongst those transcripts; ferritin-2, up-regulated in the lungstage of development (Wangwiwatsin, et al., 2020). Ferritin, the highly conserved iron storage protein, can store extensive levels of iron within its isoforms; Ferritin-2, characteristic of somatic tissues, and Ferritin-1 (Schussler, et al., 1995, Glanfield, et al., 2007). Compared to day-6 schistosomula, Ferritin-2 genes were down regulated in day-13 parasites. Although downregulated, ferritin-2 was still expressed during a time when iron should be necessary for schistosome growth and development (Wangwiwatsin, et al., 2020). In the present study, the ferritin heavy chain (TC17941) was the single most upregulated transcript in the schistosomula, within the re-annotated data set, with a fold change of 12,231.51 (Table 1). Consequently, both studies support evidence that iron is used by the schistosome for growth, development and maintenance. This research could also advocate claims that lung schistosomula are able to absorb and scavenge iron, before their gut is differentiated. It is evident that RNA-seq holds the promise of the future for bioinformatic data collection and gene expression analysis, accelerating further research in Schistosoma transcriptomics.

\section{Conclusions}

In summary, the additional annotations and pathway analysis of public domain transcriptomic data (Gobert, et al., 2009) has revealed numerous insights into the genes potentially contributing to several important functions, within the lung-stage schistosomula of S. japonicum. Enriched schistosome genes are relevant to immune evasion, energy metabolism and development, demonstrating schistosomulum adaption to the mammalian host and their environment, which could provide new interventions for disease control. The lung-stage schistosomula is recognised as 
a target of vaccine development (Gobert, et al., 2007, Wilson, 2009, Wilson, 2012), therefore targeting these enriched genes may prevent parasite development into the egg producing adult stage, responsible for host pathology and disease transmission.

\section{References:}

Abbas, W., Kumar, A.Herbein, G., 2015. The eEF1A Proteins: At the Crossroads of Oncogenesis, Apoptosis, and Viral Infections. Frontiers in Oncology 5, 75.

Alves, L. R., Oliveira, C.Goldenberg, S., 2015. Eukaryotic translation elongation factor-1 alpha is associated with a specific subset of mRNAs in Trypanosoma cruzi. BMC Microbiol. 15, 104-104.

Babu, S.Nutman, T. B., 2019. Immune Responses to Helminth infection. Clin. Immunol., 437-447. e431.

Bayer-Santos, E., Aguilar-Bonavides, C., Rodrigues, S. P., Cordero, E. M., Marques, A. F., VarelaRamirez, A., Choi, H., Yoshida, N., da Silveira, J. F.Almeida, I. C., 2013. Proteomic Analysis of Trypanosoma cruzi Secretome: Characterization of Two Populations of Extracellular Vesicles and Soluble Proteins. Journal of Proteome Research 12, 883-897.

Bennett, M. W.Caulfield, J. P., 1991. Schistosoma mansoni: Ingestion of dextrans, serum albumin, and IgG by schistosomula. Exp. Parasitol. 73, 52-61.

Billaut-Mulot, O., Schöneck, R., Fernandez-Gomez, R., Taibi, A., Capron, A., Pommier, V., PlumasMarty, B., Loyens, M.Ouaissi, A., 1994. Molecular and immunological characterization of a Trypanosoma cruzi protein homologous to mammalian elongation factor 1 gamma. Biol. Cell $82,39-44$.

Buchfink, B., Xie, C.Huson, D. H., 2015. Fast and sensitive protein alignment using DIAMOND. Nat. Methods 12, 59-60.

Chen, L., Rao, K. V. N., He, Y.-X.Ramaswamy, K., 2002. Skin-stage Schistosomula of Schistosoma mansoni Produce an Apoptosis-inducing Factor That Can Cause Apoptosis of T Cells. J. Biol. Chem. 277, 34329-34335.

Chitsulo, L., Engels, D., Montresor, A.Savioli, L., 2000. Europe PMC Funders Group The global status of schistosomiasis and its control. Acta Trop. 77, 41-51.

Colley, D. G.Secor, W. E., 2014. Immunology of human schistosomiasis. Parasite Immunol. 36, 347357.

Correnti, J. M., Brindley, P. J.Pearce, E. J., 2005. Long-term suppression of cathepsin B levels by RNA interference retards schistosome growth. Mol. Biochem. Parasitol. 143, 209-215.

Crompton, D. W., 1999. How much human helminthiasis is there in the world? J. Parasitol. 85, 397403.

Da'dara, A., Krautz-Peterson, G., Faghiri, Z.Skelly, P. J., 2012. Metabolite movement across the schistosome surface. Journal of Helminthology 86, 141-147.

de la Torre-Escudero, E., Gerlach, J. Q., Bennett, A. P. S., Cwiklinski, K., Jewhurst, H. L., Huson, K. M., Joshi, L., Kilcoyne, M., O'Neill, S., Dalton, J. P.Robinson, M. W., 2019. Surface molecules of extracellular vesicles secreted by the helminth pathogen Fasciola hepatica direct their internalisation by host cells. PLoS Negl Trop Dis 13, e0007087.

Demma, M., Warren, V., Hock, R., Dharmawardhane, S.Condeelis, J., 1990. Isolation of an abundant 50,000-dalton actin filament bundling protein from Dictyostelium amoebae. J. Biol. Chem. 265, 2286-2291. 
DosReis, G. A.Barcinski, M. A., 2001. Apoptosis and parasitism: from the parasite to the host immune response. Adv. Parasitol. 49, 133-161.

Duguet, T. B., Soichot, J., Kuzyakiv, R., Malmstrom, L.Tritten, L., 2020. Extracellular VesicleContained microRNA of $C$. elegans as a Tool to Decipher the Molecular Basis of Nematode Parasitism. Front Cell Infect Microbiol 10, 217.

El Ridi, R., Mohamed, S. H.Tallima, H., 2003. Incubation of Schistosoma mansoni Lung-Stage Schistosomula in Corn Oil Exposes Their Surface Membrane Antigenic Specificities. J. Parasitol. 89, 1064-1067.

El Ridi, R., Tallima, H., Dalton, J. P.Donnelly, S., 2014. Induction of protective immune responses against schistosomiasis using functionally active cysteine peptidases. Frontiers in genetics 5, 119-119.

Fabregat, A., Jupe, S., Matthews, L., Sidiropoulos, K., Gillespie, M., Garapati, P., Haw, R., Jassal, B., Korninger, F., May, B., Milacic, M., Roca, C. D., Rothfels, K., Sevilla, C., Shamovsky, V., Shorser, S., Varusai, T., Viteri, G., Weiser, J., Wu, G., Stein, L., Hermjakob, H.D'Eustachio, P., 2018. The Reactome Pathway Knowledgebase. Nucleic Acids Res. 46, D649-D655.

Fabregat, A., Sidiropoulos, K., Viteri, G., Forner, O., Marin-Garcia, P., Arnau, V., D'Eustachio, P., Stein, L.Hermjakob, H., 2017. Reactome pathway analysis: a high-performance in-memory approach. BMC Bioinformatics 18, 142-142.

Figueiredo, B. C. P., Ricci, N. D., de Assis, N. R. G., de Morais, S. B., Fonseca, C. T.Oliveira, S. C., 2015. Kicking in the guts: Schistosoma mansoni digestive tract proteins are potential candidates for vaccine development. Frontiers in Immunology 6, 1-7.

Fonseca, C. T., Braz Figueiredo Carvalho, G., Carvalho Alves, C.de Melo, T. T., 2012. Schistosoma Tegument Proteins in Vaccine and Diagnosis Development: An Update. Journal of Parasitology Research 2012, 1-8.

Giera, M., Kaisar, M. M. M., Derks, R. J. E., Steenvoorden, E., Kruize, Y. C. M., Hokke, C. H., Yazdanbakhsh, M.Everts, B., 2018. The Schistosoma mansoni lipidome: Leads for immunomodulation. Anal. Chim. Acta 1037, 107-118.

Glanfield, A., McManus, D. P., Anderson, G. J.Jones, M. K., 2007. Pumping iron: a potential target for novel therapeutics against schistosomes. Trends Parasitol. 23, 583-588.

Gobert, G. N., Chai, M.McManus, D. P., 2007. Biology of the schistosome lung-stage schistosomulum. Parasitology 134, 453-460.

Gobert, G. N., Moertel, L., Brindley, P. J.McManus, D. P., 2009. Developmental gene expression profiles of the human pathogen Schistosoma japonicum. BMC Genomics 10, 1-19.

Gobert, G. N., Stenzel, D. J., McManus, D. P.Jones, M. K., 2003. The ultrastructural architecture of the adult Schistosoma japonicum tegument. Int. J. Parasitol. 33, 1561-1575.

Gobert, G. N., Tran, M. H., Moertel, L., Mulvenna, J., Jones, M. K., McManus, D. P.Loukas, A., 2010. Transcriptional changes in Schistosoma mansoni during early schistosomula development and in the presence of erythrocytes. PLoS Negl Trop Dis 4, e600.

Gordon, C. A., Acosta, L. P., Gobert, G. N., Jiz, M., Olveda, R. M., Ross, A. G., Gray, D. J., Williams, G. M., Harn, D., Li, Y.McManus, D. P., 2015a. High prevalence of Schistosoma japonicum and Fasciola gigantica in bovines from Northern Samar, the Philippines. PLoS Negl Trop Dis 9, e0003108.

Gordon, C. A., McManus, D. P., Acosta, L. P., Olveda, R. M., Williams, G. M., Ross, A. G., Gray, D. J.Gobert, G. N., 2015b. Multiplex real-time PCR monitoring of intestinal helminths in humans reveals widespread polyparasitism in Northern Samar, the Philippines. Int. J. Parasitol. 45, 477483. 
Gordon, C. A., McManus, D. P., Jones, M. K., Gray, D. J.Gobert, G. N., 2016. The Increase of Exotic Zoonotic Helminth Infections: The Impact of Urbanization, Climate Change and Globalization. Adv. Parasitol. 91, 311-397.

Greenberg, R. M., 2014. Schistosome ABC multidrug transporters: From pharmacology to physiology. International Journal for Parasitology: Drugs and Drug Resistance 4, 301-309.

Gryseels, B., Polman, K., Clerinx, J.Kestens, L., 2006. Human schistosomiasis. Lancet 368, 1106-1118. Han, H., Peng, J., Gobert, G. N., Hong, Y., Zhang, M., Han, Y., Fu, Z., Shi, Y., Xu, J., Lin, J.Tao, J., 2013. Apoptosis phenomenon in the schistosomulum and adult worm life cycle stages of Schistosoma japonicum. Parasitol. Int. 62, 100-108.

Haw, R.Stein, L., 2012. Using the reactome database. Curr Protoc Bioinformatics Chapter 8, Unit8 7. Jakobsson, M. E., Malecki, J.Falnes, P. O., 2018. Regulation of eukaryotic elongation factor 1 alpha (eEF1A) by dynamic lysine methylation. RNA Biol 15, 314-319.

Jenkins, S. J., Hewitson, J. P., Jenkins, G. R.Mountford, A. P., 2005. Modulation of the host's immune response by schistosome larvae. Parasite Immunol. 27, 385-393.

Krautz-Peterson, G., Camargo, S., Huggel, K., Verrey, F., Shoemaker, C. B.Skelly, P. J., 2007. Amino Acid Transport in Schistosomes. J. Biol. Chem. 282, 21767-21775.

Krautz-Peterson, G., Simoes, M., Faghiri, Z., Ndegwa, D., Oliveira, G., Shoemaker, C. B.Skelly, P. J., 2010. Suppressing Glucose Transporter Gene Expression in Schistosomes Impairs Parasite Feeding and Decreases Survival in the Mammalian Host. PLoS Path. 6, e1000932-e1000932.

Maizels, R. M., Smits, H. H.McSorley, H. J., 2018. Modulation of Host Immunity by Helminths: The Expanding Repertoire of Parasite Effector Molecules. Immunity 49, 801-818.

Malone, J. H.Oliver, B., 2011. Microarrays, deep sequencing and the true measure of the transcriptome. BMC Biol. 9, 34.

Martin-Duran, J. M.Egger, B., 2012. Developmental diversity in free-living flatworms. Evodevo 3, 7.

McManus, D. P., Bergquist, R., Cai, P., Ranasinghe, S., Tebeje, B. M.You, H., 2020. Schistosomiasisfrom immunopathology to vaccines. Semin Immunopathol 42, 355-371.

Morales, M. E., Rinaldi, G., Gobert, G. N., Kines, K. J., Tort, J. F.Brindley, P. J., 2008. RNA interference of Schistosoma mansoni cathepsin $\mathrm{D}$, the apical enzyme of the hemoglobin proteolysis cascade. Mol. Biochem. Parasitol. 157, 160-168.

Peng, J., Gobert, G. N., Hong, Y., Jiang, W., Han, H., McManus, D. P., Wang, X., Liu, J., Fu, Z., Shi, Y.Lin, J., 2011. Apoptosis Governs the Elimination of Schistosoma japonicum from the NonPermissive Host Microtus fortis. PLoS ONE 6, e21109-e21109.

Peng, J., Yang, Y., Feng, X., Cheng, G.Lin, J., 2010. Molecular characterizations of an inhibitor of apoptosis from Schistosoma japonicum. Parasitol. Res. 106, 967-976.

Retra, K., deWalick, S., Schmitz, M., Yazdanbakhsh, M., Tielens, A. G., Brouwers, J. F.van Hellemond, J. J., 2015. The tegumental surface membranes of Schistosoma mansoni are enriched in parasite-specific phospholipid species. Int. J. Parasitol. 45, 629-636.

Riengrojpitak, S., Anderson, S.Wilson, R. A., 1998. Induction of immunity to Schistosoma mansoni: interaction of schistosomula with accessory leucocytes in murine skin and draining lymph nodes. Parasitology 117 ( Pt 4), 301-309.

Schistosoma japonicum Genome Sequencing Functional Analysis Consortium, 2009. The Schistosoma japonicum genome reveals features of host-parasite interplay. Nature 460, 345351.

Schussler, P., Potters, E., Winnen, R., Bottke, W.Kunz, W., 1995. An isoform of ferritin as a component of protein yolk platelets in Schistosoma mansoni. Mol. Reprod. Dev. 41, 325-330.

Sepulveda, J., Tremblay, J. M., DeGnore, J. P., Skelly, P. J.Shoemaker, C. B., 2010. Schistosoma mansoni host-exposed surface antigens characterized by sera and recombinant antibodies from schistosomiasis-resistant rats. Int. J. Parasitol. 40, 1407-1417. 
Skelly, P. J., Da'dara, A. A., Li, X.-H., Castro-Borges, W.Wilson, R. A., 2014. Schistosome Feeding and Regurgitation. PLoS Path. 10, e1004246-e1004246.

Skelly, P. J., Tielens, A. G.Shoemaker, C. B., 1998. Glucose Transport and Metabolism in Mammalianstage Schistosomes. Parasitology today (Personal ed.) 14, 402-406.

Tan-ariya, P., Buathong, S., Leelayoova, S.Mungthin, M., 2014. Role of Cathepsin B in Schistosoma japonicum Infection. Tropical Medicine and Parasitology 37, 43-53.

Timm, T., Annoscia, G., Klein, J.Lochnit, G., 2017. The Eukaryotic Elongation Factor 1 Alpha (eEF1 $\alpha$ ) from the Parasite Leishmania infantum Is Modified with the Immunomodulatory Substituent Phosphorylcholine (PC). Molecules 22, 2094-2094.

Toh, S. Q., Glanfield, A., Gobert, G. N.Jones, M. K., 2010. Heme and blood-feeding parasites: friends or foes? Parasit Vectors 3, 108.

Toh, S. Q., Gobert, G. N., Malagon Martinez, D.Jones, M. K., 2015. Haem uptake is essential for egg production in the haematophagous blood fluke of humans, Schistosoma mansoni. FEBS J. 282, 3632-3646.

Wang, S., Zhang, Z., Wang, Y., Gadahi, J. A., Xu, L., Yan, R., Song, X.Li, X., 2017. Toxoplasma gondii Elongation Factor 1-Alpha (TgEF-1 $\alpha)$ Is a Novel Vaccine Candidate Antigen against Toxoplasmosis. Frontiers in Microbiology 8, 168.

Wangwiwatsin, A., Protasio, A. V., Wilson, S., Owusu, C., Holroyd, N. E., Sanders, M. J., Keane, J., Doenhoff, M. J., Rinaldi, G.Berriman, M., 2020. Transcriptome of the parasitic flatworm Schistosoma mansoni during intra-mammalian development. PLoS Negl Trop Dis 14, e0007743.

Wilson, R. A., 2009. The saga of schistosome migration and attrition. Parasitology 136, 1581-1592. Wilson, R. A., 2012. Virulence factors of schistosomes. Microbes Infect 14, 1442-1450.

Yang, F., Demma, M., Warren, V., Dharmawardhane, S.Condeelis, J., 1990. Identification of an actinbinding protein from Dictyostelium as elongation factor 1a. Nature 347, 494-496.

Zerda, K. S., Dresden, M. H.Chappell, C. L., 1988. Schistosoma mansoni: expression and role of cysteine proteinases in developing schistosomula. Exp. Parasitol. 67, 238-246.

Zhu, L., Liu, J., Dao, J., Lu, K., Li, H., Gu, H., Liu, J., Feng, X.Cheng, G., 2016. Molecular characterization of $S$. japonicum exosome-like vesicles reveals their regulatory roles in parasite-host interactions. Scientific Reports 6, 25885-25885. 